\title{
Central venous access ports in the interventional radiology suite - one-centre experience
}

\author{
Bartosz Zabicki ${ }^{A, B, C, D, E, F, G}$, Nattakarn Limphaiboo $\left.\right|^{B, D, E, F}$, Marte Johanne Veilemand Holstad ${ }^{B, D, E, F,}$, \\ Katarzyna Perkowska ${ }^{B, D, E, F}$ \\ Department of Diagnostic and Interventional Radiology, Poznan University of Medical Sciences, Poznan, Poland
}

\section{Abstract}

Purpose: Central venous access ports (CVAP) are widely used to provide long-term vascular access for the delivery of chemotherapeutic medications. The aim of this study was to evaluate the clinical outcomes and complications following CVAP implantation in the interventional radiology suite.

Material and methods: A retrospective analysis was conducted on 937 oncology patients who underwent CVAP implantation between January 2009 and June 2017. Information regarding patient characteristics, operative data, and procedural outcomes was collected and analysed.

Results: A total of 937 patients scheduled for CVAP were included in the final analysis. Initial success was achieved in 930 patients (99.3\%), and overall completion was achieved in 933 patients (99.6\%). There were 63 complications overall. Among these, 19 (2.0\%) occurred during the intra- and perioperative period and 44 were late complications (4.7\%). No CVAP-related mortalities were observed.

Conclusions: The analysis in the present study revealed that the CVAP is a safe and effective route for long-term administration of chemotherapy with an overall complication rate of $6.7 \%$ throughout the entire device duration. The CVAP implantation procedure carried out in the interventional radiology suite provides an advantage in the management of procedural, vascular and catheter-related complications.

Key words: central venous access ports, chemotherapy administration, intraoperative complications, postoperative complications.

\section{Introduction}

Intensive methods used in the administration of chemotherapeutic medications has increased the need for implantable devices that provide long-term vascular access. The significant venous toxicity of chemotherapeutic drugs often results in the rapid termination of peripheral access [1]. Central venous access ports (CVAP) are associated with reduced venous toxicity during prolonged courses of chemotherapy, playing an essential role in improving the patient's quality of life in modern oncology [2-4]. This retrospective study analyses the experience of the CVAP implantation procedure in a large sample of oncology patients, considering procedural success rate, complications and their management.

\section{Material and methods}

\section{Patient population}

Between January 2009 and June 2017, 937 patients underwent CVAP implantation in the Department of Diagnostic and Interventional Radiology at Poznan University of Medical Sciences. A total of 940 CVAP implantation

Correspondence address:

Bartosz Zabicki, Poznan University of Medical Sciences, University Hospital no. 1, 1/2 Dluga St., 61-848 Poznan, Poland, phone: +48601678238,

e-mail: bzabicki@gmail.com

Authors' contribution:

A Study design · B Data collection · C Statistical analysis · D Data interpretation · E Manuscript preparation · F Literature search · G Funds collection 
procedures were performed in 937 oncology patients. Inclusion criteria for this study were that all patients had documented active malignancy and had CVAP implanted for chemotherapy administration. Detailed demographic data are shown in Table 1. All patients were treated with intravenous chemotherapy as a mainstay or adjunctive therapy in primary neoplastic diseases, or presented metastatic lesions. The most common diagnosis was colorectal cancer (48.3\%), followed by breast (25.3\%), gastric (7.3\%), and pancreatic carcinoma (4.5\%). Difficulties concerning peripheral venous access were the initial primary indication for implantation. Later, CVAP implantation became a standard procedure in all patients scheduled for longterm systemic chemotherapy.

\section{Data analysis}

Information regarding the age, gender, site of primary tumour, operative data, complications, and outcome of the implantation procedure within the patient population was retrospectively collected. The follow-up period for this study for each patient lasted from the date of CVAP insertion until the date of scheduled device removal, death, or the last recorded date. Initial success of the procedure was defined as one resulting in maintained aspiration and infusion throughout the system and properly healed surgical wounds at the time of the second session of chemotherapy administration. Overall completion rate describes the number of patients who had CVAP successfully implanted, despite any initial procedure failure. Any complication occurring within the period before the second chemotherapy session was defined as an early complication, while complications occurring after the second chemotherapy session were defined as late complications.

\section{Central venous access ports placement procedure}

CVAP placement procedures were performed in the interventional radiology suite. A single model of port was used in all cases (Celsite, B. Braun, Boulogne-Billancourt, France). Each patient received oral premedication with $7.5 \mathrm{mg}$ of midazolam and $1 \mathrm{~g}$ of prophylactic IV cefazolin prior to the procedure. The second dose of cefazolin was administered 12 hours after the first dose. The subclavian vein (SCV) was the initial choice of vascular access for introducing the port. The internal jugular vein (IJV) or brachiocephalic vein (BCV) was chosen if unsuccessful attempts at subclavian puncture occurred, or if SCV stenosis, compression or occlusion was suspected, based on anamnesis and clinical examination. Direct puncture using the Seldinger method was performed according to anatomical landmarks, mostly without ultrasound guidance or pre-procedural imaging of the central veins.

CVAP were subcutaneously implanted under local anaesthesia. A single-lumen catheter was inserted through the guide wire at the puncture site and the tip was placed
Table 1. Patient characteristics and diagnosis

\begin{tabular}{|l|c|}
\hline Characteristic & 937 \\
\hline Number of patients & \\
\hline Age, years & $58.6(12.8)$ \\
\hline Mean (SD) & $19-82$ \\
\hline Range & \\
\hline Gender, $n$ (\%) & $542(57.8)$ \\
\hline Female & $395(42.2)$ \\
\hline Male & \\
\hline Primary malignancy, $n(\%)$ & $453(48.3)$ \\
\hline Colorectal & $237(25.3)$ \\
\hline Breast & $68(7.3)$ \\
\hline Gastric & $42(4.5)$ \\
\hline Pancreatic & $37(3.9)$ \\
\hline Bladder & $28(3.0)$ \\
\hline Lung & $19(2.0)$ \\
\hline Prostate & $14(1.5)$ \\
\hline Sarcoma MSK & $12(1.3)$ \\
\hline Gall bladder & $8(0.9)$ \\
\hline Testicular & $6(0.6)$ \\
\hline Lymphoma & $3(0.3)$ \\
\hline Oesophageal & $3(0.3)$ \\
\hline Ovarian & $2(0.2)$ \\
\hline Small intestine & $2(0.2)$ \\
\hline Kidney & $1(0.1)$ \\
\hline Skin & $1(0.1)$ \\
\hline Brain & $1(0.1)$ \\
\hline Thymoma & \\
\hline
\end{tabular}

at the level of the cavoatrial junction, confirmed under fluoroscopy. The pouch for the port reservoir was dissected into the subcutaneous tissue of the chest wall. The catheter was tunnelled under the skin from the pouch to the venous entry site and subsequently inserted into the vena cava superior. The port reservoir was then immobilised with sutures in the subcutaneous tissue. Initially, the catheter length was precisely measured on fluoroscopy and cut to the correct length in the pouch. Later, a technique consisting of aligning the catheter with a properly positioned guidewire was developed, allowing the proper measurement of the required catheter length and preparation of a suitable catheter with the port outside of the body. Afterwards the entire system was inserted at once, which made the procedure quicker and more convenient. After the subcutaneous dissolvable sutures had been placed, post-procedural digital subtraction angiography (DSA) was performed to evaluate port integrity and post-operative flow through the system. Finally, the reservoir was aspirated and flushed with a heparinised saline solution. 
A chest X-ray was carried out up to 12 hours after implantation to confirm the correct position of the CVAP and the catheter tip, and to rule out possible complications such as pneumothorax.

\section{Complication management}

Early complications such as pneumothorax were managed with catheter drainage in symptomatic cases or when the pneumothorax was larger than $3 \mathrm{~cm}$ on the chest X-ray. The CVAP placement procedure was discontinued in cases when the patient's general condition was poor, prior to liquid transfusions, blood pressure normalisation and medical symptom management. Inadvertent arterial puncture was a minor issue, which was not found to be a true complication. It was satisfactorily treated with short-term compression of the punctured area. Inadvertent sheath insertion into the brachiocephalic artery in one case prompted its immediate removal with occlusion balloon control and preparation of a covered stent in case of uncontrolled bleeding (Figure 1). Bleeding from a ruptured internal thoracic artery occurred in one case in which the sheath was inadvertently removed. This was managed with balloon occlusion and subsequent implantation of a coronary covered stent (Figure 2). Early infection was another indication for port removal and subsequent antibiotic therapy.

The management of late complications, such as catheter occlusion, was carried out using the "pull-and-push" technique with saline infusion until the proper flow was restored. Angiography was performed when port malfunction was suspected. In cases concerning disturbed infusion, the immobilised catheter tip was snared through the femoral access site, and subsequently released and repositioned away from the fibrin sheath (Figure 3). A case of pinch-off syndrome and catheter rupture was managed with endovascular therapy. The loose catheter fragment in this case was snared and removed through the femoral access site. Another CVAP implantation was carried out using the BCV access site. DVT complications were managed with anticoagulation. Port infection was managed initially with antibiotic infusions through the port system, and the ports were scheduled for removal in cases in which symptom relief was not achieved.

\section{Ethics}

In accordance with Polish law and good clinical practice (GCP) regulations, this retrospective analysis did not require approval of the Bioethics Committee of Poznan University of Medical Sciences. The methods described in this article were carried out in accordance with the guidelines and regulations of our institution. Informed consent was acquired from all patients before the procedure.

\section{Data availability statement}

All data generated or analysed during this study are included in this published article (and its Supplementary Information files).

\section{Results}

Primary technical success of CVAP placement was achieved in $99.3 \%(930 / 937)$ of patients. In 7 patients,

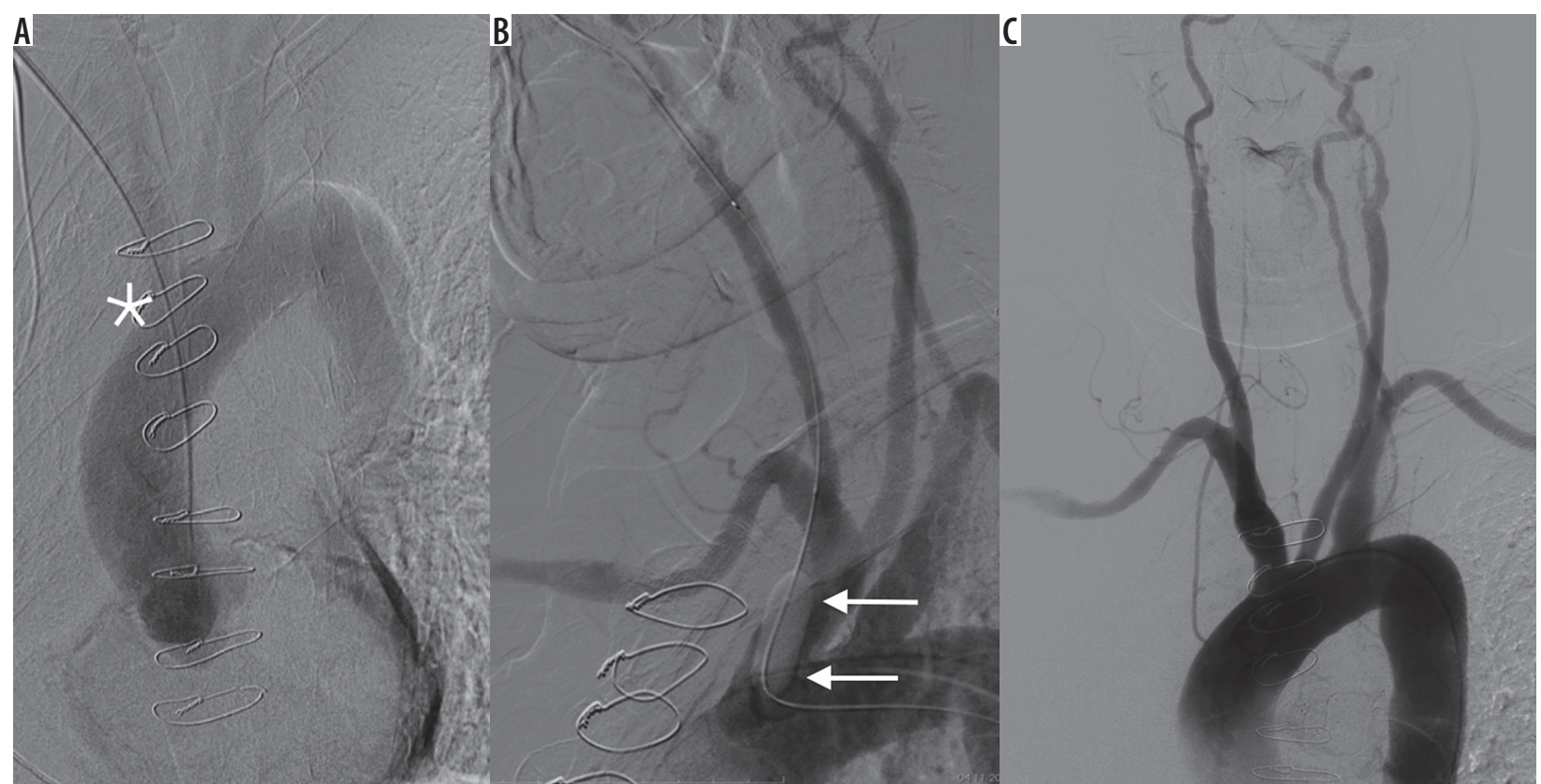

Figure 1. A) Peel-away sheath inadvertently inserted into the ascending aorta through the brachiocephalic artery (asterisk). B) External compression together with balloon catheter (arrows) to control the vascular injury site after sheath removal. C) Control digital subtraction angiography - no neurologic complications, no extravasation, patency of the arteries 


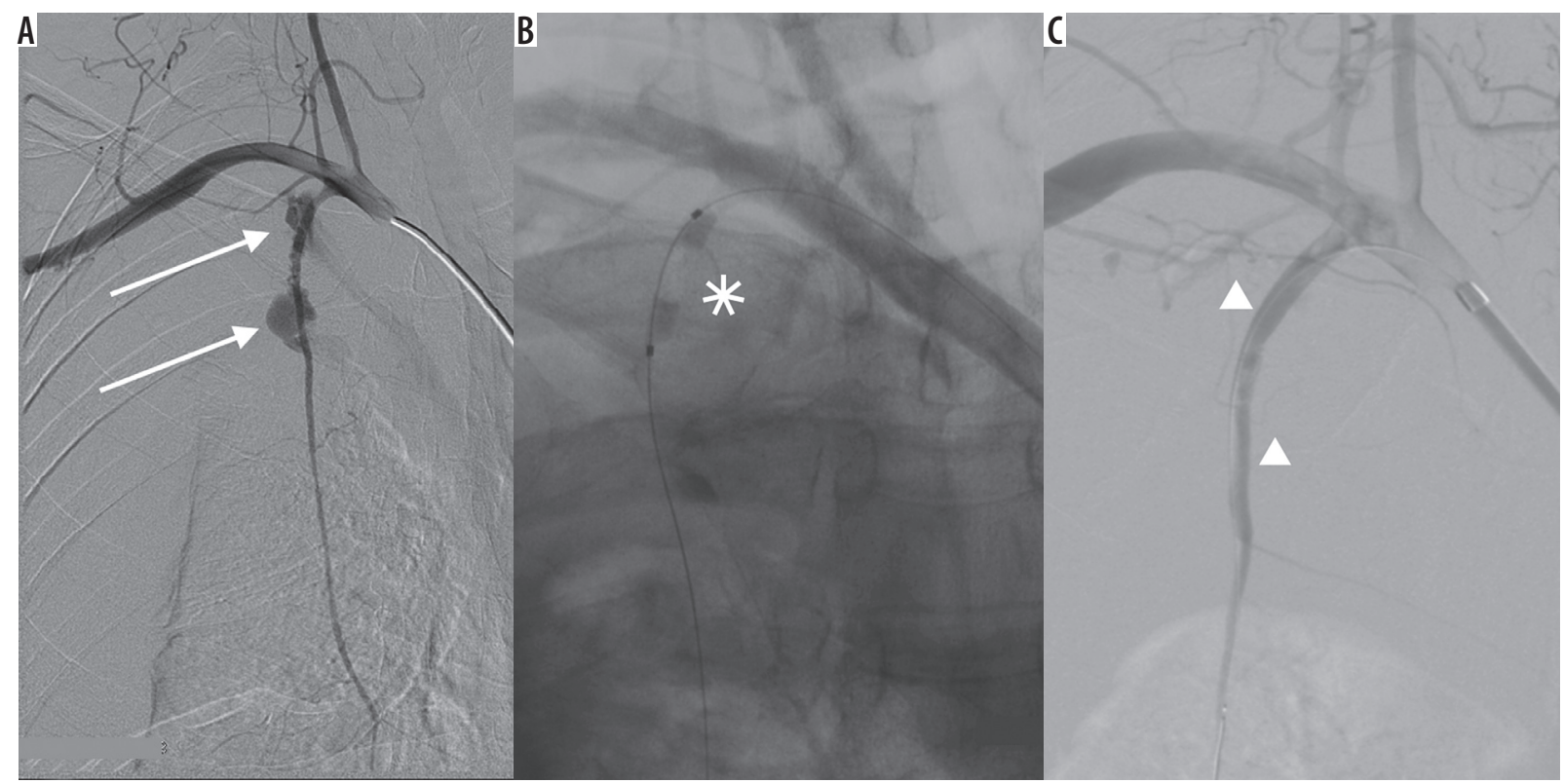

Figure 2. A) Digital subtraction angiography of right subclavian artery. Ruptured right internal thoracic artery in 2 spots (arrows) with massive bleeding. B) Temporary bleeding control with angioplasty balloon catheter (asterisk). C) Final extravasation control with coronary covered stents (arrowheads)
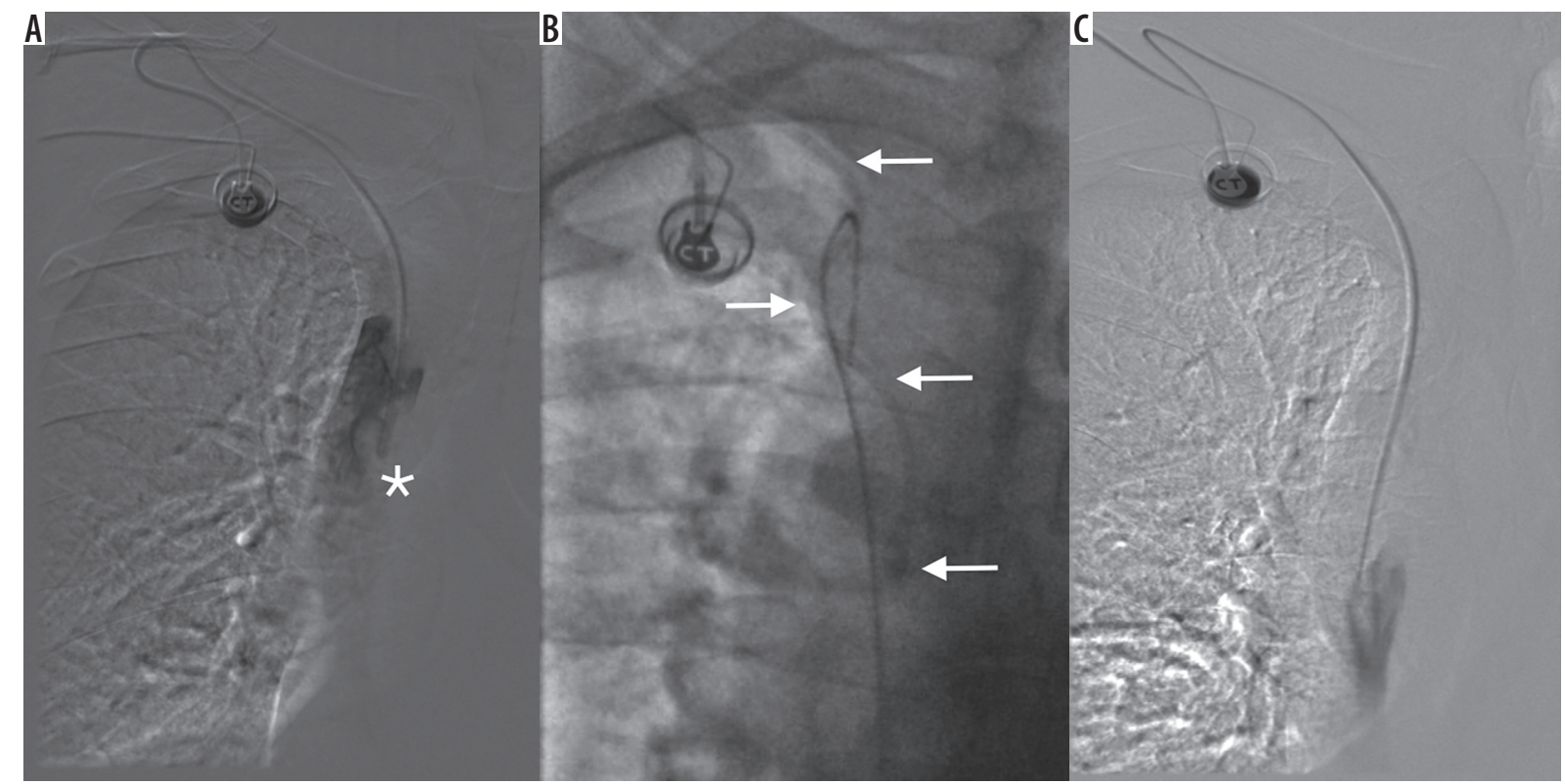

Figure 3. A) Port malfunction with disturbed aspiration and altered injection/infusion performance. Tip of the catheter (asterisk) located in the "fibrin sheath" in vena cava superior. B) Port catheter (arrows) captured from femoral vein access site. C) Reposition of the port catheter. Control angiography undisturbed, free flow through the port system

initial implantation failure occurred. In 3 cases, the patients' poor general condition prompted discontinuation of the procedure (vasovagal syncope, nausea/vomiting, allergic reaction to the prophylactic antibiotic) that prevented completion of the procedure. In two cases, inadvertent arterial puncture and sheath insertion occurred. In one of these cases, the sheath was removed carelessly, resulting in massive bleeding from the ruptured internal thoracic artery, which led to haemothorax and circulatory shock. The complication was treated endovascularly and the patient fully recovered. In the other case, the sheath was removed safely with angiographic control using endovascular techniques for guidance, causing no bleeding. The two patients technically had successful implantations, but the ports had to be removed within a few days due to signs of infections. Three of the patients with initial port implantation failure underwent successive port implantation procedures. The overall completion rate was $99.6 \%$ (933/937) (Table 2).

The access route used for implantation was the SCV in 839 patients, the IJV in 40 patients and the BCV in 60 patients. Initial subclavian access was unsuccessful in 
Table 2. Details of central venous access ports placement

\begin{tabular}{|l|c|}
\hline Parameter & 940 \\
\hline Total number of port procedures & \\
\hline Insertion site, $n$ (940) (\%) & $839(89.3)$ \\
\hline Subclavian vein & $61(6.5)$ \\
\hline Brachiocephalic vein & $40(4.3)$ \\
\hline Internal jugular vein & $804(85.5)$ \\
\hline Right side & $136(14.5)$ \\
\hline Left side & $21.7(4.2)$ \\
\hline Procedure time, minutes & $17-80$ \\
\hline Mean (SD) & \\
\hline Range & 11 months \\
\hline Known port duration & 4 days - 88 months \\
\hline Mean & \\
\hline Range & $930 / 937$ (99.3) \\
\hline Completion rate & $933 / 937$ (99.6) \\
\hline Primary technical success & \\
\hline Overall completion rate & \\
\hline
\end{tabular}

Table 3. Early and late complications

\begin{tabular}{|l|c|}
\hline Early complications (out of 940) & No. (\%) \\
\hline Pneumothorax & $12(1.3)$ \\
\hline Arterial rupture and massive bleeding & $1(0.1)$ \\
\hline Inadvertent arterial sheath placement & $1(0.1)$ \\
\hline Clinical status disabling procedure completion & \\
\hline Vasovagal syncope & $1(0.1)$ \\
\hline Vomiting, nausea & $1(0.1)$ \\
\hline $\begin{array}{l}\text { Nausea, hypotension, allergic reaction to prophylactic } \\
\text { antibiotic }\end{array}$ & $1(0.1)$ \\
\hline Early port infection & $2(0.2)$ \\
\hline Total & $19 / 940(2.0)$ \\
\hline Late complications (out of 933) & No. (\%) \\
\hline Catheter occlusion & $13(1.4)$ \\
\hline Deep venous thrombosis & $7(0.8)$ \\
\hline Port infection & $7(0.8)$ \\
\hline Catheter malfunction & $16(1.7)$ \\
\hline Catheter rupture & $1(0.1)$ \\
\hline Total & $44 / 933(4.7)$ \\
\hline Total complications & $63(6.7)$ \\
\hline
\end{tabular}

18 (1.9\%) patients. For these patients, CVAP were successfully implanted via the IJV or the BCV. Eighty-five percent of ports were placed on the right side of the chest. The mean surgical time was 21.7 minutes (standard devia- tion 4.2 minutes). The average time during which a CVAP remained in place was 11 months (range: 4 days to 88 months). Details concerning the placement procedure are shown in Table 2.

CVAP complications developed in 63 implantation procedures (6.7\%). Early complications comprised pneumothorax, followed by inadvertent arterial puncture, arterial sheath insertion, poor general condition, and early port infection (Table 3). No air embolism, early catheter dislocation, or port dysfunction occurred. Five out of 12 cases of pneumothorax necessitated tube thoracostomy and drainage, and the rest were treated conservatively due to small volume and lack of progression.

Late complications, as summarised in Table 3, occurred in $4.7 \%$ (44/933) of implanted patients and included port infection, port malfunction, deep venous thrombosis and catheter occlusion, dislocation or rupture. In 16 cases, aspiration was disturbed and the quality of the injections was variously compromised. All of the ports were found to be intact and patent upon angiographic investigation. In 3 cases in which infusion was essentially compromised, endovascular repositioning of the port catheter was performed and proper flow was successfully restored.

Catheter occlusion was encountered in 13 cases (1.4\%). All of them were recanalised using the "push and pull" injection technique with saline under fluoroscopic control.

In one case, catheter rupture and dislocation (socalled pinch-off) was observed, in which the catheter was dislodged into the right ventricle. The loose catheter was removed via snaring from the femoral vein access site, and the patient received a new port catheter through the brachiocephalic access site.

Seven patients presented with symptoms of port infection. In two of them, intravenous antibiotics and direct port infusions were effective. Five patients required removal of the port, and two of them had a new port implanted in the later phase of treatment. Symptomatic DVT was encountered in 7 cases, and anticoagulation therapy was sufficient to relieve the symptoms in all cases.

\section{Discussion}

CVAP improve the quality of life in patients by avoiding multiple peripheral vein punctures during prolonged chemotherapeutic treatment. CVAP have a decreased risk of causing extravasation of chemotherapeutic agents compared to peripheral access, which may cause local damage to tissue and thrombosis and thrombophlebitis depending on the agent administered (e.g. 5-FU, an inflammatory substance). This is especially true concerning prolonged therapy, as the risk of extravasation increases over time with peripheral line infusions [2, 3, 5]. CVAP are also indicated for the administration of parenteral nutrition, 
volume replacement, detoxification, and in the diagnostic evaluation and monitoring of patients [6]. Newer ports (called power ports or computed tomography-compatible ports) also resist high flow contrast injections when used in diagnostic imaging [7].

Placement of the catheter can be achieved through multiple routes. The most common access sites used are the IJV or SCV $[4,8,9]$. Ultrasound-guided puncture and surgical cut-down access through the cephalic vein at the deltoid-pectoralis groove are well-established alternatives for implantation [6].

The SCV was the initial site of all planned CVAP implantations due to better cosmetic results and less limitation of movement compared to the IJV [10]. Studies have reported an advantage of a shorter access time and lower risk of infection in long-term use associated with the SCV access site $[5,11,12]$. The anatomic-landmark-guided technique was used in this study when using the SCV access site. This was because the ultrasound-guided catheter placement through the SCV access site can be complicated due to its anatomical location, compared to the IJV. Bony structures were therefore used as landmarks during the implantation instead of US guidance, which is the standard operating procedure in some societies $[4,13]$. Eightyfive percent of CVAP were placed on the right side of the chest in this study. The side of venous access was determined by the presence of unilateral breast carcinoma, the site of planned or completed irradiation, ulcerations on the chest wall, previous surgery or implanted pacemaker, structural anomalies, pre-existing unilateral venous thrombosis, or other circumstances related to personal preference $[4,6,9]$.

Procedural complications occurring during or shortly after the implantation of a CVAP are mainly dependent on the patient status, method of implantation and local vascular anatomy. In this study, periprocedural complications occurred in 19 cases out of the 937 performed (2\%).

The most common intra-operative complication was pneumothorax (1.3\%). The incidence of inadvertent arterial puncture and pneumothorax experienced in this study was consistent with the risk rates described in other studies in which anatomical-landmark-guided techniques and subclavian vein access sites were used $[9,14]$. Different technical complications included failed puncture of the initially planned vein, prior to subsequent successful attempts at cannulation. Other complications, including haematoma, dyspnoea and vasovagal syncope, occurred less frequently $(0.7 \%$ in total).

Infection and mechanical complications represent frequent and important issues that accompany the insertion of CVAP in cancer patients, primarily due to the nature of their condition. Proper training of the nurses perform- ing infusions significantly decreases the infection rate [15, 16]. Several of the patients presenting with signs of infection were treated with antibiotics initially. When this did not relieve the symptoms, the patients were scheduled for port removal with later re-implantation. An immunocompromised state, in addition to the prothrombotic and endothelial-damaging effect of the malignancies and chemotherapy, is an important risk factor. Deep venous thrombosis presented with ipsilateral pain and swelling in the arm (7 patients). The most commonly observed late complication in the present study was catheter occlusion, occurring in 13 patients (1.4\%).

Port implantation can be performed by surgeons, anaesthesiologists or interventional radiologists, with a comparable degree of procedural success $[6,17]$. In this study, all procedures were performed in the interventional radiology suite, where additional equipment and radiological expertise offered an advantage in the management of complications. Imaging modalities allowed for accurate catheter placement, short implantation time and the possibility of intravascular repositioning of catheters and recanalisation of intravenous access.

In recent retrospective studies, complication rates ranged from $1.4 \%$ to $11.1 \%$ (intra-operative) and from $6.5 \%$ to $17.1 \%$ (post-operative) $[2,4,18]$. The complication rates are comparable in this study, although a lower rate of complications in the late post-operative period was observed. Variables such as patient body mass index (BMI), venous access site and catheter tip location have been controversially discussed to be predicting factors for complication risk $[2,4,16]$. Observations made by other studies also revealed pneumothorax, infection, thrombosis and catheter occlusion as common complications during CVAP implantation $[2,4,6]$.

\section{Conclusions}

The analysis in the present study revealed that CVAP is a safe and effective route for long-term administration of chemotherapy with an overall complication rate of $6.7 \%$ throughout the entire device duration. The average time for which the port was in place was approximately 11 months, and the vast majority of devices remained in situ and complication-free until death of the patient or end of the treatment. The CVAP implantation procedure carried out in the interventional radiology suite provides an advantage in the management of procedural, vascular, and catheter-related complications.

\section{Disclosure}

The authors declare no conflict of interests. 


\section{References}

1. Cheung E, Baerlocher MO, Asch M, Myers A. Venous access: a practical review for 2009. Can Fam Physician 2009; 55: 494-6.

2. Ignatov A, Hoffman $\mathrm{O}$, Smith $\mathrm{B}$, et al. An 11-year retrospective study of totally implanted central venous access ports: complications and patient satisfaction. Eur J Surg Oncol 2009; 35: 241-6.

3. Granic M, Zdravkovic D, Krstajic S, et al. Totally implantable central venous catheters of the port-a-cath type: complications due to its use in the treatment of cancer patients. J BUON 2014; 19: 842-6.

4. Mudan S, Giakoustidis A, Morrison D, et al. 1000 Port-A-Cath placements by subclavian vein approach: single surgeon experience. World J Surg 2015; 39: 328-34.

5. Kreidieh F, Moukadem HA, El Saghir NS. Overview, prevention and management of chemotherapy extravasation. World J Clin Oncol 2016; 7: 87-97.

6. Teichgräber UK, Pfitzmann R, Hofmann HAF. Central venous port systems as an integral part of chemotherapy. Dtsch Arztebl Int 2011; 108: 147-154

7. Goltz JP, Noack C, Petritsch B, Kirchner J, Hahn D, Kickuth R. Totally implantable venous power ports of the forearm and the chest: initial clinical experience with port devices approved for high-pressure injections. Br J Radiol 2012; 85: 966-972.

8. Taxbro K, Berg S, Hammarskjöld F, Hanberger H, Malmvall BE. A prospective observational study on 249 subcutaneous central vein access ports in a Swedish county hospital. Acta Oncol 2013; 52: 893-901.

9. Karamustafaoglu YA, Yagci S, Kocal A, Yoruk Y. Comparison of Implantable Central Venous Ports: Subclavian Versus Jugular Access. J Clin Anal Med 2013; 4: 495-498.
10. Capaccioli L, Nistri M, Distante V, Rontini M, Manetti A, Stecco A. Insertion and management of long-term central venous devices: role of radiologic imaging techniques. Radiol Med 1998; 96: 369-374.

11. Ruesch S, Walder B, Tramèr MR. Complications of central venous catheters: internal jugular versus subclavian access - a systematic review. Crit Care Med 2002; 30: 454-460.

12. Timsit JF. What is the best site for central venous catheter insertion in critically ill patients? Critical Care 2003; 7: 397-399.

13. Orsi F, Grasso RF, Arnaldi P. et al. Ultrasound guided versus direct vein puncture in central venous port placement. J Vasc Access 2000; 1: 73-77.

14. Tsotsolis N, Tsirgogianni K, Kioumis I, et al. Pneumothorax as a complication of central venous catheter insertion. Ann Transl Med 2015; 3: 40.

15. Kornbau C, Lee KC, Hughes GD, Firstenberg MS. Central line complications. Int J Crit Illn Inj Sci 2015; 5: 170-178.

16. Seok JP, Kim YJ, Cho HM, Ryu HY, Hwang WJ, Sung TY. A retrospective clinical study: complications of totally implanted central venous access ports. Korean J Thorac Cardiovasc Surg 2014; 47: 26-31.

17. Ozyuvaci E, Kutlu F. Totally implantable venous access devices via subclavian vein: A retrospective study of 368 oncology patients. Adv Therapy 2006; 23: 574.

18. Matsushima H, Adachi T, Iwata T, et al. Analysis of the outcomes in central venous access port implantation performed by residents via the internal jugular vein and subclavian vein. J Surg Educ 2016; 74: 443-449. 